\title{
Application of Dual-Frequency Ultrasound to Radiation-Induced Fibrosis in a Breast Cancer Patient
}

\author{
Yean Su Choi \\ Eun Soo Park
}

Department of Plastic \& Reconstructive Surgery, College of Medicine, Soonchunhyang Bucheon University Hospital , Bucheon, Korea
Received December 14, 2017

Accepted December 17, 2017

\section{Correspondence}

Eun Soo Park

Department of Plastic \& Reconstructive Surgery, College of Medicine, Soonchunhyang Bucheon University Hospital, 170 Jomaru-ro, Wonmi-gu, Bucheon 14584, Korea

Tel.: +82-32-621-5319

Fax: +82-32-621-5662

E-mail: peunsoodschmc.ac.kr

(C) Korean Society for Laser Medicine and Surgery

(c) This is an open access article distributed under the terms of the Creative Commons Attribution NonCommercial License (http://creativecommons.org/ licenses/by-nc/4.0) which permits unrestricted noncommercial use, distribution, and reproduction in any medium, provided the original work is properly cited.
Radiation-induced fibrosis is a well-known complication of postmastectomy radiotherapy. Patients suffering from radiation-induced fibrosis usually complain of pain, skin thickening, muscle atrophy, and limited range of motion. A few studies suggested various methods to treat the symptoms caused by fibrosis, but approved ones are rare. This paper introduces a remarkable case of the treatment of a 47-year-old female patient who had pain and discomfort feeling on her right breast after post-mastectomy radiotherapy. Treatments using dual-frequency ultrasound (LDM-MED ${ }^{\circledR}$ ) were applied to the patient actively for 2 months, and the patient's symptoms improved significantly. In this case, the effectiveness and possibility of LDM treatments were checked as a prominent management option of radiation-induced fibrosis.

\section{Key words}

Radiation-induced fibrosis; Dual-frequency ultrasound 


\section{INTRODUCTION}

In many breast cancer patients, radiation therapy is usually followed after breast conservation surgery to reduce recurrence and to prolong survival as a result. As the radiation therapy has prolonged patients' life successfully, its long-term complication has been revealed. While there are only local skin reaction including local irritation and drying within the first 2 months, late side effects involve more serious states such as fibrosis, sclerodermatous changes, skin telangiectasia, atrophy, skin necrosis and secondary malignancies. ${ }^{1,2}$ Especially, radiationinduced fibrosis (RIF) is one of the most common major late effect which can occur 4 to 12 months after radiation therapy. Its symptoms are various according to patients from pain, lymphedema, skin induration and thickening to muscle atrophy, limited range of motion, and ulceration. ${ }^{3}$ These symptoms can be worsening with time and years damaging patients' quality of life. Recently published case reported that low level light therapy has positive effect of preventing fibrosis in patients under radiotherapy. ${ }^{4}$ However, it is not generalized yet and cannot be the method of treating fibrosis that already occurred. We introduce our successful case of RIF, which was improved notably after applying dual-frequency ultrasound therapy on her breast.

\section{CASE REPORT}

\section{Patient and method}

A 47-year-old female patient had breast cancer combine operation of surgery department and plastic surgery department 3 years ago. After quadrantectomy was completed to remove ductal carcinoma in situ on her right breast, latissimus dorsi myocutaneous flap was selected to reconstruct it. Patient discharged on postoperative day 8 without any specific complications including seroma, hematoma, wound dehiscence, and flap loss. According to normal breast conservation surgery protocol, radiation therapy had been applied for 2 months. The patient
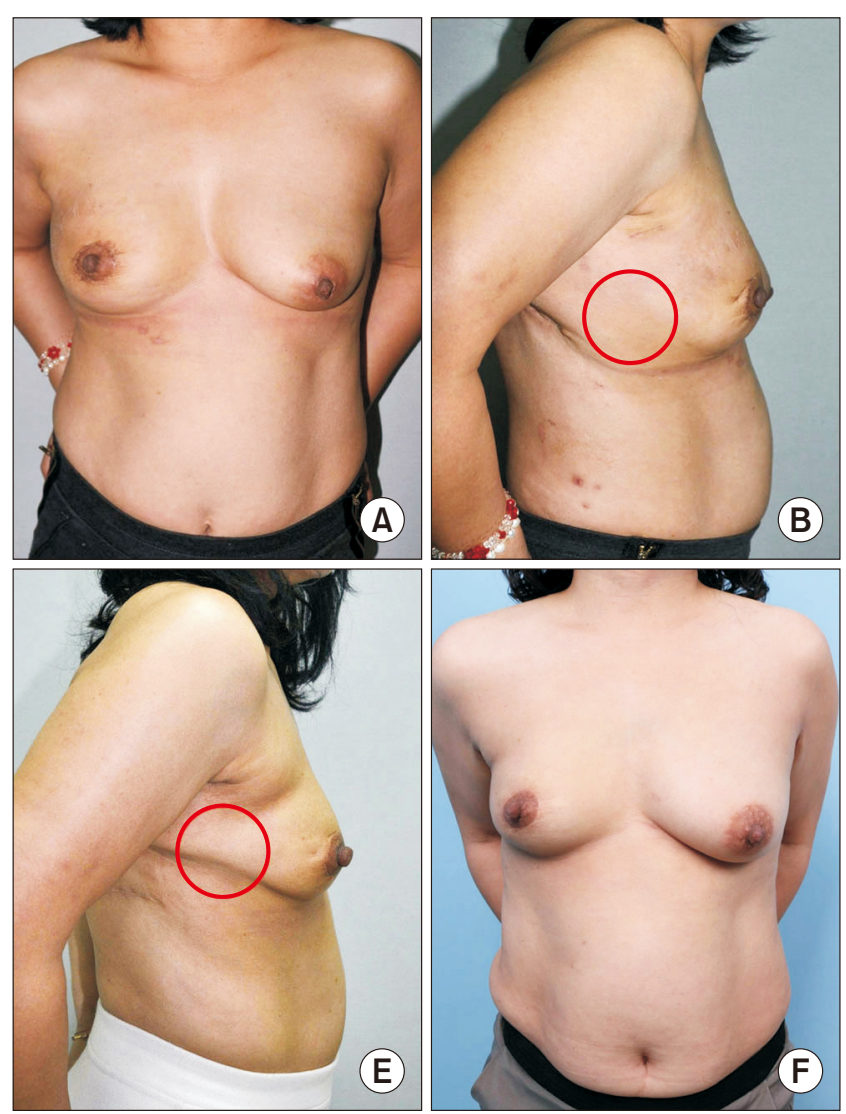
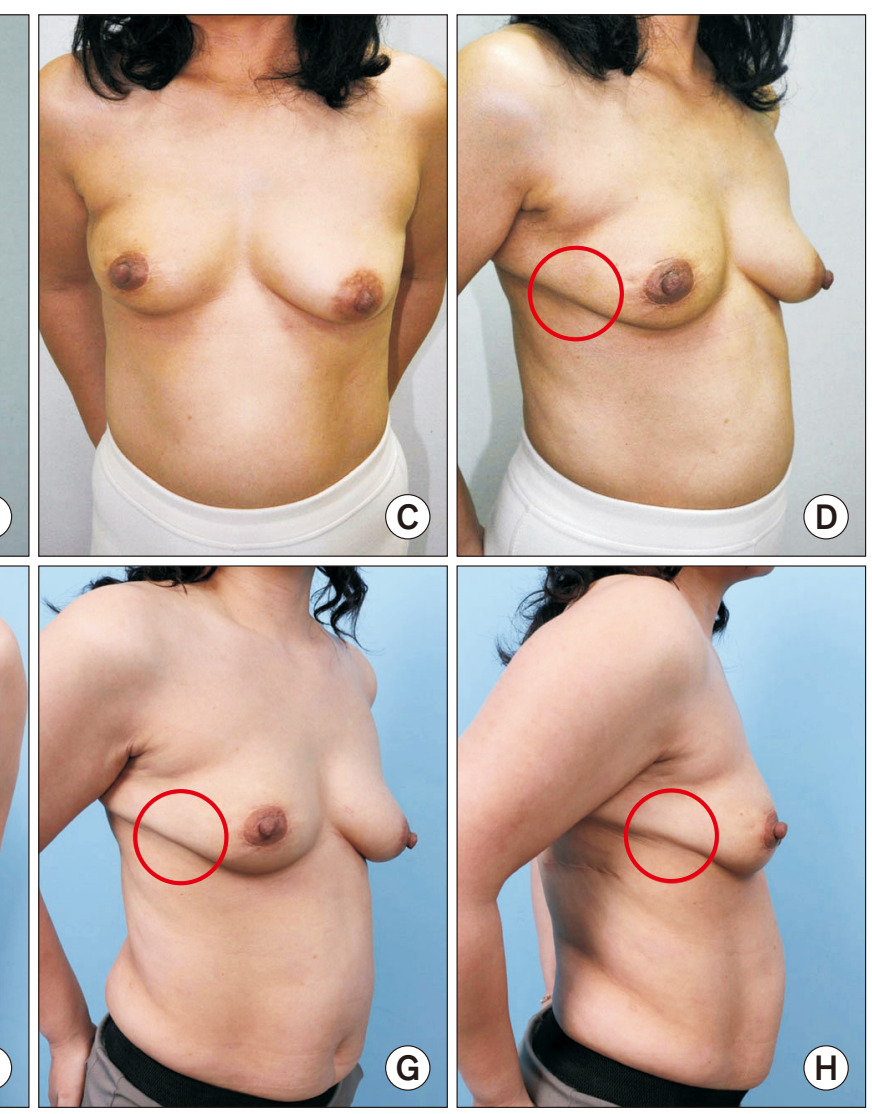

Fig. 1. 47-year-old female was suffered from radiation-induced fibrosis on her right breast. She was diagnosed as ductal carcinoma in situ of right breast. Radiotherapy had been performed since 1 month after mastectomy and reconstruction operation. Dual-frequency ultrasound (LDM) treatments was applied to relieve the complications of radiotherapy. (A, B) Before radiotherapy. (C-E) 9 months after the last radiotherapy. (F-H) 1 year after the last LDM treatment. 
tolerated radiation treatment well, however, side effects appeared on operation site. She complained about discomfort and pain on her right breast as well as contour deformity of lateral side of the breast, and these symptoms didn't disappear until 1 year passed. To relieve it, we considered LDM-MED ${ }^{\circledR}$ (Wellcomet, Karlsruhe, Germany) as effective non-invasive method which can soften fibrosis.

LDM treatments had been performed total 12 times for 2 months, 1-2 times a week on average. Protocol was set as $10 \mathrm{MHz}$ with intensity of $1.5 \mathrm{~W} / \mathrm{cm}^{2}$ and $1.0 \mathrm{~W} / \mathrm{cm}^{2}$ for 2 minutes each, then was followed by $3 / 10 \mathrm{MHz}$ dual frequency setting with intensity of $1.0 \mathrm{~W} / \mathrm{cm}^{2}$ and $2.0 \mathrm{~W} /$ $\mathrm{cm}^{2}$ each for 3 minutes. During each treatment, the patient's right breast, especially the lateral side where the symptom is most prominent was treated for 7 minutes. Ultrasonic gel was used as coupling medium during all treatments.

Follow up continued every 2 or 3 months after the last LDM treatment. We did physical examinations and asked about patient's opinion to evaluate whether the symptoms was improved. Ultrasonography was also followed once a year by surgery department, so we could compare radiologic findings of the breast before and after the LDM treatments.

\section{Result}

The outcome of LDM treatments was remarkable more than our expectation. At the end of the second LDM treatment, the patient already felt relief of the symptoms, especially pain was decreased from NRS score 6 to 2 .
Although it is subjective opinion, the patient was also very satisfied with contour of the breast which looks more natural (Fig. 1). We could also find improvements in radiologic findings of the patient's right breast. Comparing ultrasonography images which were taken before and 1 year after the LDM treatments, skin thickening of the area above the latissimus dorsi myocutaneous flap decreases from $4 \mathrm{~mm}$ to $2 \mathrm{~mm}$, which means it became normal. In addition, premammary fat became more hypoechoic with regression of haziness making clear border between skin and fat layer (Fig. 2).

\section{DISCUSSION}

With increasing numbers of women who select breast conservation surgery to treat their breast cancer, radiotherapy became common treatment option. While traditional indications of radiotherapy included tumor size of $5 \mathrm{~cm}$ or larger, involvement of 4 or more lymph nodes, and extension to skin of muscle, current irradiation is offered to patients with smaller tumor size and fewer lymph nodes involvement. ${ }^{5,6}$ So managing side effects of radiotherapy is the key issues we are facing.

RIF is one of the most important late effect which can contribute to patient morbidity. It may occur in any organs in the irradiation field including skin and soft tissue, lung, as well as gastrointestinal and genitourinary tracts. Increased radiation dose and hypofractionation, and prolongation of therapy are known as the factors which can increase the risk of RIF. ${ }^{7}$ Recently, a few studies reported that specific genetics are associated with RIF. In breast
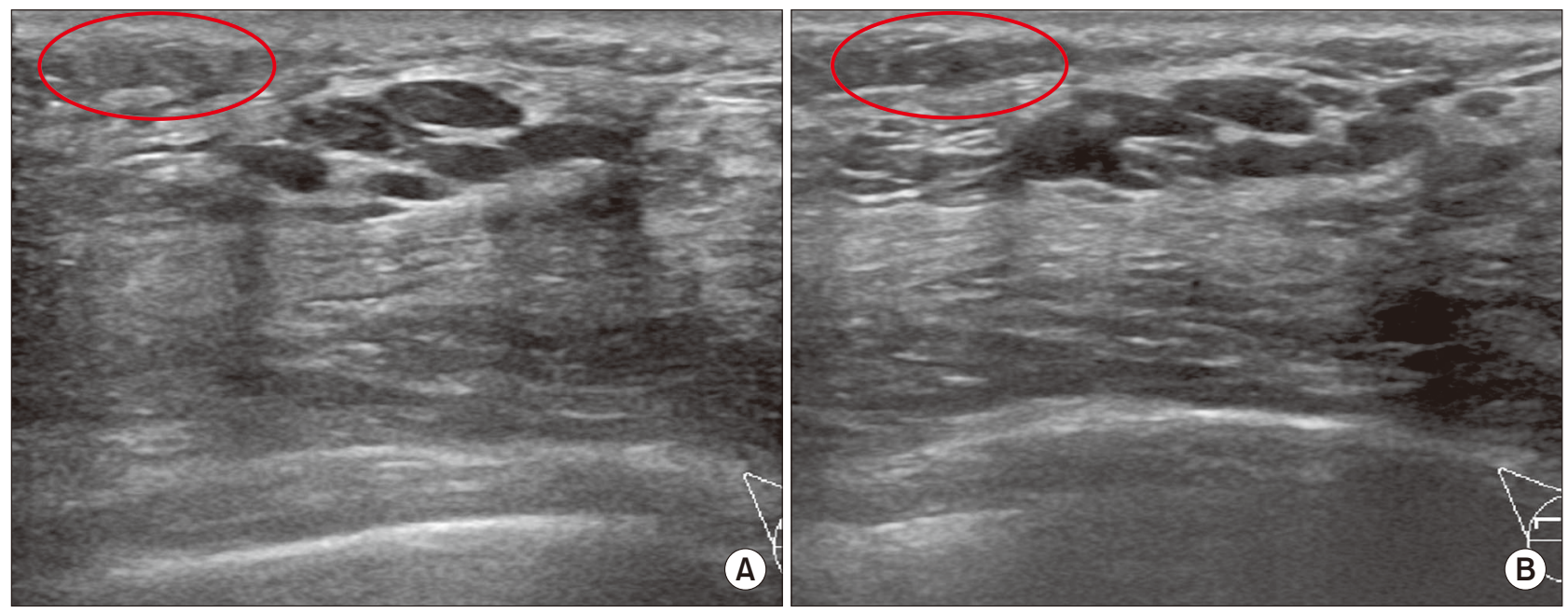

Fig. 2. Ultrasonography images of the patient's right breast. Skin thickening of the area above the latissimus dorsi myocutaneous flap decreases from $4 \mathrm{~mm}$ to $2 \mathrm{~mm}$. Premammary fat became more hypoechoic with regression of haziness making clear border between skin and fat layer. (A) Before LDM treatment. (B) 1 year after the last LDM treatment. 
cancer, for instance, genetic variation of the ataxia-telangiectasia mutated (ATM) gene increase risk of RIF after postmastectomy radiotherapy. ${ }^{8}$ RIF shows specific features as fibroblasts proliferation, myofibroblast differentiation, and synthesis of collagen, proteoglycans and extracellular matrix. ${ }^{9.10}$ Radiation induces DNA damage and inflammation which stimulate transforming growth factor $\beta 1$ (TGF- $\beta 1$ ) activity resulting in fibrosis mechanism. ${ }^{11}$ Considering the pathophysiology of RIF, many clinical trials have been performed to find out effective treatment. Although a few studies of antioxidant medication with pentoxifylline and vitamin $E$ are shown to improve tissue compliance in patients with RIF, only few therapies for RIF are approved. ${ }^{12,13}$

Scar tissue and fibrosis can induce pain, muscle spasm, and joint contracture. To increase viscoelasticity and extensibility of tissue, ultrasound is often applied in previous studies. ${ }^{14,15}$ Based on therapeutic effects of ultrasound on pain, we decided to perform treatments using LDM-MED $^{\circledR}$, the dual frequency ultrasound. In our case, effect on pain and discomfort feeling was revealed soon. She felt more comfortable when moving her right arm and palpating her right breast herself after the second LDM treatment was finished. The patient has experienced significant improvement of symptoms since the last treatment until now, more than a year from then. In addition, objective evaluation using ultrasonography images confirmed improvements of soft tissue. Radiologic findings implied that quality of premammary fat changed better as well as skin thickening became normal.

Meaningful point of our study is more effective and faster result of treatments than other previously reported cases. Especially, the degree of satisfaction mentioned by the patient was really high. As the number of women who received post-mastectomy radiation therapy (PMRT) increasing, the importance of solving the problems associated with radiation such as fibrosis is also increased. Through the successful management of this case, we checked the possibility of LDM treatments as a new treatment option for patients suffered from RIF. We are planning to apply LDM treatments to other patients who are complaining similar symptoms. Collected data from these patients will help us to establish more effective and trustful management of RIF using dual frequency ultrasound.

\section{REFERENCES}

1. Cheah NL, Wong DW, Chetiyawardana AD. Radiation-induced morphea of the breast: a case report. J Med Case Rep 2008;2:136.
2. de Giorgi V, Santi R, Grazzini M, Papi F, Gori A, Rossari S, et al. Synchronous angiosarcoma, melanoma and morphea of the breast skin 14 years after radiotherapy for mammary carcinoma. Acta Derm Venereol 2010;90:283-6.

3. Dörr W, Hendry JH. Consequential late effects in normal tissues. Radiother Oncol 2001;61:223-31.

4. Seité S, Bensadoun RJ, Mazer JM. Prevention and treatment of acute and chronic radiodermatitis. Breast Cancer (Dove Med Press) 2017;9:551-7.

5. Frasier LL, Holden S, Holden T, Schumacher JR, Leverson G, Anderson B, et al. Temporal trends in postmastectomy radiation therapy and breast reconstruction associated with changes in national comprehensive cancer network guidelines. JAMA Oncol 2016;2:95-101.

6. Recht A, Comen EA, Fine RE, Fleming GF, Hardenbergh PH, Ho AY, et al. Postmastectomy radiotherapy: an American Society of Clinical Oncology, American Society for Radiation Oncology, and society of surgical oncology focused guideline update. J Clin Oncol 2016;34:4431-42.

7. Johansson S, Svensson H, Denekamp J. Dose response and latency for radiation-induced fibrosis, edema, and neuropathy in breast cancer patients. Int J Radiat Oncol Biol Phys 2002;52:1207-19.

8. Andreassen CN, Overgaard J, Alsner J, Overgaard M, Herskind C, Cesaretti JA, et al. ATM sequence variants and risk of radiation-induced subcutaneous fibrosis after postmastectomy radiotherapy. Int J Radiat Oncol Biol Phys 2006;64:776-83.

9. Wynn TA. Cellular and molecular mechanisms of fibrosis. J Pathol 2008;214:199-210.

10. Wynn TA. Fibrotic disease and the $T(H) 1 / T(H) 2$ paradigm. Nat Rev Immunol 2004;4:583-94.

11. Straub JM, New J, Hamilton CD, Lominska C, Shnayder Y, Thomas SM. Radiation-induced fibrosis: mechanisms and implications for therapy. J Cancer Res Clin Oncol 2015;141:198594.

12. Jacobson G, Bhatia S, Smith BJ, Button AM, Bodeker K, Buatti $J$. Randomized trial of pentoxifylline and vitamin E vs standard follow-up after breast irradiation to prevent breast fibrosis, evaluated by tissue compliance meter. Int J Radiat Oncol Biol Phys 2013;85:604-8.

13. Patel V, McGurk M. Use of pentoxifylline and tocopherol in radiation-induced fibrosis and fibroatrophy. Br J Oral Maxillofac Surg 2017;55:235-41.

14. Robertson VJ, Baker KG. A review of therapeutic ultrasound: effectiveness studies. Phys Ther 2001;81:1339-50.

15. Morishita K, Karasuno H, Yokoi Y, Morozumi K, Ogihara H, Ito T, et al. Effects of therapeutic ultrasound on range of motion and stretch pain. J Phys Ther Sci 2014;26:711-5. 